\title{
Construction d'un modèle de répartition des arbres par classes de grosseur pour des plantations d'épicéa commun (Picea abies L Karst) en Ardenne belge
}

\author{
$P$ Lejeune
}

\begin{abstract}
Unité de gestion et économie forestières, Faculté des sciences agronomiques de Gembloux, passage des Déportés, 2, B-5030 Gembloux, Belgique
\end{abstract}

(Reçu le 28 janvier 1993; accepté le 23 août 1993)

\begin{abstract}
Résumé - La construction d'un modèle de répartition d'arbres par classes de grosseur pour des peuplements d'épicéa commun (Picea abies $L$ Karst) a été envisagée au départ de 141 placettes de 10 ares. L'influence de la distribution théorique (comparaison des distributions normale et Weibull) et de la méthode d'estimation des paramètres (pour Weibull) ont été analysés. Malgré une plus grande flexibilité de la distribution de Weibull, son utilisation ne conduit pas à une plus grande précision du modèle. La faiblesse des effectifs des échantillons utilisés semble être la cause principale de l'imprécision fournie par les différents modèles testés. Sur la base des données utilisées, la distribution normale, plus simple à mettre en œuvre, a été préférée pour la construction du modèle de répartition des grosseurs, pour les peuplements d'épicéa commun.
\end{abstract}

épicéa / modèle de répartition / distribution normale / distribution de Weibull

Summary - Construction of a tree-size distribution model for Norway spruce (Picea abies L Karst) plantations in the Belgian Ardennes. The construction of a girth distribution model for Norway spruce (Picea abies $L$ Karst) plantations has been considered using 141 sample plots of 1000 $\mathrm{m}^{2}$. The effects of the theoretical distribution (comparison of the normal and Weibull distributions) and the esimation methods have been analysed. Despite the higher flexibility of the Weibull distribution, its use does not lead to a more accurate prediction of the distribution. The small number of samples measured in the plots seems to be the primary cause of the inaccuracy of the various models. Considering the data analysed, the normal distribution, which is easier to use, is proving to be more suitable for the creation of distribution model for such stands.

spruce / distribution model / normal distribution / Weibull distribution 


\section{INTRODUCTION}

Malgré les évolutions importantes, observées au niveau des techniques de modélisation dans le domaine forestier (Houllier et al, 1991), les modèles de type peuplement, qui sont apparus les premiers (Munro, 1974), connaissent encore de nos jours une grande popularité auprès des forestiers.

Ils sont présentés généralement sous la forme de tables de production qui décrivent l'évolution au cours du temps de variables globales (volume par hectare, nombre de tiges par hectare, circonférence moyenne, hauteur dominante,...) pour des peuplements équiennes et monospécifiques, en fonction du niveau de fertilité (site index) et éventuellement du type de sylviculture (Dagnelie et al, 1988).

Le caractère synthétique des informations présentes dans ces tables constitue cependant un des inconvénients majeurs de ce genre d'outil. La dimension et plus particulièrement la grosseur des arbres est en effet déterminante quant aux possibilités d'utilisation de ces produits. La connaissance de la répartition des tiges d'un peuplement par classes de grosseur est donc une information très précieuse tant pour le gestionnaire forestier que pour l'industriel devant s'assurer un approvisionnement en produits ligneux de dimensions bien définies.

Une amélioration technique permet de pallier cette carence. II s'agit de créer un modèle de répartition des tiges par classes de grosseur, utilisant comme variables explicatives certains paramètres descriptifs du peuplement, fournis par la table de production (tels que circonférence moyenne, site index [il s'agit de la hauteur dominante supposée atteinte à l'âge de 50 ans et qui est fonction de la hauteur dominante observée et de l'âge du peuplement (Dagnélie et al, 1988)], âge...).
Plusieurs approches permettent d'obtenir un tel modèle (Cao et Burkhart, 1984 ; Hyink et Moser, 1983; Rennols et al, 1985 ; Borders et al, 1987). La plus courante consiste à utiliser une fonction de densité de probabilité pour représenter la répartition des individus constituant le peuplement, en classes de grosseur (Knoebel et al, 1986).

L'objectif de cette étude est d'analyser les différentes étapes de construction et de validation d'un tel modèle pour des plantations d'épicéa commun (Picea abies $\mathrm{L}$ Karst) en Ardenne belge.

Nous présenterons d'abord la méthode de construction du modèle, en évoquant les problèmes liés au choix de la distribution théorique (voir p 54), à l'estimation des paramètres (voir p 56) et à l'utilisation d'un test d'appréciation de la qualité du modèle (voir $p$ 57). Nous décrirons ensuite les données utilisées pour cette étude (voir $p$ 58). Les résultats de nos analyses seront détaillés $p$ 58. Un exemple concret d'application du modèle de répartition des tiges sera proposé (voir p 63) avant de tirer quelques conclusions (voir p 64).

\section{CONSTRUCTION D'UN MODELLE DE RÉPARTITION DE TIGES}

\section{Choix d'une distribution}

De nombreuses distributions théoriques ont été utilisées pour caractériser la structure de peuplements forestiers. Parmi les principales, il convient de citer les distributions normale, log-normale, gamma, beta, Sb de Johnsson et Weibull (Borders et al, 1987). Nous avons choisi de comparer les performances de la distribution de Weibull, qui constitue la référence dans ce genre d'applications, et la distribution normale dont la mise en œuvre est très 
simple, dans un modèle de répartition de tiges.

\section{Distribution de Weibull}

La distribution de Weibull est celle qui depuis une vingtaine d'années connait et continue de connaître le plus de succès, essentiellement pour 2 raisons (Bailey et Dell, 1973) : une grande flexibilité et l'existence d'une forme explicite de sa fonction de répartition. Compte tenu des moyens de calcul disponibles actuellement, ce dernier argument n'a cependant plus beaucoup de valeur.

La fonction de densité de probabilité et la fonction de répartition de la distribution de Weibull sont décrites ci-dessous (équations [1] et [2]). Les 3 paramètres apparaissant dans ces relations sont respectivement:

a) le paramètre de localisation, donnant la valeur minimum de la distribution ;

b) le paramètre d'échelle ;

c) le paramètre de forme, qui détermine la dissymétrie de la distribution, celle-ci étant gauche ou droite selon que $c$ est supérieur ou inférieur à 3,6. Pour une valeur de $c=1$ la distribution prend l'allure d'une exponentielle décroissante ; pour une valeur de 3,6 celle d'une distribution normale.

$f(x)=\frac{c}{b}\left(\frac{x-a}{b}\right)^{c-1} \exp \left[-\left(\frac{x-a}{b}\right)\right]^{c}$

pour $x \geq a, a \geq 0, b>0, c>0$

$=0$, sinon

$F(x)=1-\exp \left[-\left(\frac{x-a}{b}\right)\right]$

La figure 1 donne un aperçu de la forme que peut revêtir une telle distribution en fonction des valeurs prises par les paramètres.

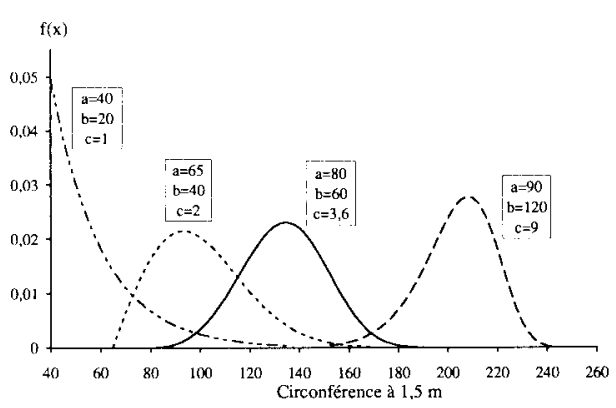

Fig 1. Représentation graphique de la fonction de Weibull pour différentes valeurs des paramètres $a, b$ et $c$.

\section{Distribution normale}

La distribution normale est moins fréquemment utilisée dans la construction de modèles de répartition des grosseurs d'arbres. Elle est caractérisée par une forme unimodale symétrique qui ne permet pas une aussi grande flexibilité que la distribution de Weibull. Gérard (1975) et Rondeux (1973) I'ont cependant utilisé pour caractériser la distribution des tiges de peuplements d'épicéa commun issus de plantations.

La fonction de densité de probabilité et la fonction de répartition de cette distribution correspondent aux équations [3] et [4].

$$
\begin{gathered}
f(x)=\frac{1}{\sigma 2 \pi} \exp \left[-\frac{1}{2}\left(\frac{x-m}{\sigma}\right)^{2}\right] \\
F(x)=\int_{-\infty}^{x} f(x) d x
\end{gathered}
$$

Cette distribution est définie par 2 paramètres qui sont $m$, la moyenne arithmétique, et $\sigma$, l'écart type de la population. 


\section{Estimation des paramètres}

II est important de distinguer dans le processus de construction d'un modèle de répartition des grosseurs de tiges, la phase d'estimation des paramètres et la phase de prédiction des paramètres.

La phase d'estimation consiste à calculer par une méthode adaptée les para-
La méthode du maximum de vraisemblance consiste en la résolution de manière itérative d'un système de 3 équations à 3 inconnues (équations [5], [6] et [7]) (Jonhson et Kotz, 1970).

$$
\hat{b}=\left[n^{-1} \sum_{i=1}^{n}\left(x_{i}-\hat{a}\right)^{\hat{c}}\right]^{c-1}
$$

$$
\hat{c}=\left[\left(\sum_{i=1}^{n}\left(x_{i}-\hat{a}\right)^{\hat{c}} \log \left(x_{i}-\hat{a}\right)\left(\sum_{i=1}^{n}\left(x_{i}-\hat{a}\right) \hat{c}\right)^{-1}-n^{-1} \sum_{i=1}^{n} \log \left(x_{i}-\hat{a}\right)\right]-1\right.
$$

mètres d'une distribution théorique définie pour un échantillon de population donné. Cette opération est répétée pour un certain nombre de placettes d'échantillonnage représentatives de situations aussi diverses que possible et les paramètres ainsi définis sont mis en relation (par régression) avec des variables caractérisant le peuplement.

La prédiction des paramètres est l'opération qui consiste à utiliser ces relations pour définir les paramètres d'une distribution qui servira à établir la répartition supposée des tiges du peuplement auquel on s'intéresse.

L'estimation des paramètres d'une distribution de Weibull peut s'avérer difficile (Zarnoch et Dell, 1985). Plusieurs démarches existent, dont la méthode du maximum de vraisemblance qui est la plus utilisée et qui nécessite d'importants calculs itératifs. D'autres approches, plus simples, font appel aux percentiles (Johnson et Kotz, 1970) ou aux moments non centrés (Burk et Newberry, 1984). Une méthode plus récente utilise les moments pondérés (Grender et al, 1990).

Nous nous limiterons dans cette étude à la comparaison des méthodes du maximum de vraisemblance, des moments non centrés et des moments pondérés.

$$
\hat{(c-1)} \sum_{i=1}^{n}\left(x_{i}-\hat{a}\right)^{-1}=\hat{c} \hat{b}-\hat{c} \sum_{i=1}^{n}\left(x_{i}-\hat{a}\right)^{\hat{c}-1}
$$

avec $n$, l'effectif de l'échantillon ; $x_{i}$, la circonférence de l'arbre i.

La méthode des moments non centrés est proposée par Burk et Newberry (1984) qui ont construit un système de 3 équations à 3 inconnues basé sur les 3 premiers moments non centrés de la distribution des circonférences (équations [8], [9] et [10]. Ce système doit également être résolu de manière itérative.

$$
\begin{gathered}
\mu_{3}^{\prime}=\hat{b}^{3}\left(\Gamma_{3}-3 \Gamma_{1} \Gamma_{2}+2 \Gamma_{1}{ }^{3}\right)+3 \mu_{1}^{\prime} \mu_{2}^{\prime}-2\left(\mu_{1}{ }^{\prime}\right)^{3} \\
\hat{b}=\sqrt{\frac{\mu_{2}{ }^{\prime}-\left(\mu_{1}\right)^{2}}{\Gamma_{2}-\Gamma_{1}{ }^{2}}} \\
\hat{a}=\mu_{1}{ }^{\prime}-\hat{b} \Gamma_{1}
\end{gathered}
$$

avec $\mu_{k}{ }^{\prime}=\sum_{i=1}^{n} x_{i}^{k}$, et $\Gamma_{k}=\Gamma\left(1+\frac{k}{\hat{c}}\right)$

où $\Gamma($.) est la fonction gamma.

La méthode des moments pondérés se différencie de la précédente par le fait que les moments qui y sont utilisés donnent un poids plus important à la partie droite de la 
distribution devant être représentée (correspondant aux arbres de grosses dimensions). L'équation [11] donne une définition théorique des moments pondérés à droite, alors que la relation [12] permet de calculer ces mêmes moments dans le cas d'échantillons dont les observations sont classées par ordre décroissant de grosseurs. L'estimation des paramètres $a, b$ et $c$ découle alors de la résolution du système constitué des relations [13], [14] et [15].

$$
\left.M_{l, j}=\int_{0}^{1} \mathrm{x}(\mathrm{F})^{\prime} \cdot \mathrm{F}(\mathrm{x})\right)^{j} \cdot \mathrm{dF}(\mathrm{x})
$$

où $M_{l, j}$ est le moment d'ordre / et de degré $j ; \mathrm{x}(\mathrm{F})$, la forme inverse de la fonction de répartition $F(x)$.

$$
\hat{M}_{l, j}=\frac{1}{n} \cdot \sum_{i=1}^{n-j} x_{[i]}^{l} \cdot \frac{C_{j}^{i-1}}{C_{j}^{n-1}}
$$

où $x_{[i]}$ est la ie observation de l'échantillon classé par ordre décroissant de grosseurs et

$$
C_{j}^{i}=\frac{j !}{(j-i) ! i !}
$$

$$
\begin{gathered}
\hat{\mathrm{c}}=\frac{\ln (2)}{\ln \left[\frac{2 M_{1,1}-M_{1,0}}{2 \cdot\left(5 M_{1,1}-M_{1,0}-6 M_{1,2}+2 M_{1,3}\right.}\right]} \\
\hat{\mathrm{a}}=\frac{4\left[M_{1,0} \cdot\left(3 M_{1,2}-M_{1,3}-M_{1,1}\right)-M_{1,1}^{2}\right.}{M_{1,0}-8 M_{1,1}+12 M_{1,2}-4 M_{1,3}} \\
\hat{\mathrm{b}}=\frac{M_{1,0}-\hat{\mathrm{a}}}{1} \\
\Gamma\left(1+\frac{\hat{\Lambda}}{\hat{\mathrm{c}}}\right)
\end{gathered}
$$

Les trois méthodes d'estimation des paramètres de la distribution de Weibull ont été intégrées dans un programme informatique (Weib3) écrit en basic et fonctionnant sur PC.

L'estimation des paramètres d'une distribution normale s'effectue sans problème. II s'agit en effet de la moyenne et de l'écart type estimés de la population qui sont donnés par les relations [16] et [17].

$$
\hat{m}=-\frac{1}{n} \sum_{i=1}^{n} x_{i}
$$

$$
\hat{\sigma}=\sqrt{\frac{\sum_{i=1}^{n}\left(x_{i}-\hat{m}\right)^{2}}{n-1}}
$$

\section{Appréciation de la qualité du modèle de répartition des grosseurs}

II est important de pouvoir apprécier si la distribution théorique que l'on utilise donne une bonne représentation de la distribution des tiges d'un peuplement. Ce test de conformité peut être utilisé à différents stades de la construction du modèle de répartition. Au moment de l'estimation des paramètres, il est nécessaire de tester la concordance entre les distributions théoriques et observées au niveau de chaque placette. On aura ainsi une idée de l'aptitude de la famille de distribution choisie à représenter le type de peuplements concerné par le modèle. Ce test de conformité est surtout appliqué lors de l'utilisation finale du modèle lorsque les paramètres de la distribution théorique sont prédits à partir de variables descriptives du peuplement.

L'utilisation des tests de conformité classiquement utilisés en statistique pose cer- 
tains problèmes. Les tables de valeurs critiques relatives au test de KolmogorovSmirnov ne prévoient pas le cas de distribution telle que Weibull où 3 paramètres doivent être estimés (Dagnelie, 1968). L'utilisation du test $\chi^{2}$ de Pearson impose, quant à lui, de regrouper certaines classes extrêmes en cas d'effectifs insuffisants (Dagnelie, 1975).

Nous avons finalement appuyé nos comparaisons sur l'utilisation d'un indice créé par Reynolds et al (1988), qui correspond à la sommation des différences absolues entre les effectifs prédits et observés au sein de classes de grosseur définies pour chaque distribution. Ces différences sont en outre pondérées par le volume des individus représentés dans les 2 distributions (équation [18]). Cet indice peut être exprimé de manière relative en le divisant par le volume total correspondant à la distribution observée (équation [19]).

$$
\begin{gathered}
e=N \sum_{j=1}^{k}\left|\int_{l_{j}} w(x) d \hat{F}(x)-\right| \int_{l_{j}} w(x) d F^{*}(x) ![18] \\
e^{\prime}=\frac{e}{N \int w(x) d F^{*}(x)}
\end{gathered}
$$

où $N$ est l'effectif total ; $k$, le nombre de classes; $l_{j}$, la je classe; $w(x)$, le facteur de pondération (ici le volume individuel); $\hat{F}(x)$, la fonction de répartition de la distribution estimée; $F^{\star}(x)$, la fonction de répartition de la distribution observée.

\section{DESCRIPTION DU MATÉRIEL EXPÉRIMENTAL}

Nous utilisons dans cette étude les données relatives à 141 placettes de 10 ares relevant d'une expérimentation destinée à comparer différentes modalités d'échantillonnage. Ces placettes ont été implantées de manière pseudo-aléatoire dans divers peuplements purs d'épicéa commun âgés de 28 à 110 ans et traités en futaie régulière (Laurent et Rondeux, 1982). Le tableau I reprend les principales caractéristiques dendrométriques des peuplements échantillonnés.

\section{RÉSULTATS}

\section{Estimation des paramètres}

Nous avons estimé, pour les 141 placettes disponibles, les paramètres $a, b$ et $c$ de la distribution de Weibull par les 3 méthodes décrites p 55, à l'aide du programme Weib3. Sauf dans quelques cas d'échantillons de faibles effectifs, les procédures

Tableau I. Principales caractéristiques dendrométriques relatives aux placettes d'échantillonnage utilisées.

\begin{tabular}{lcccc} 
Variables & Moyenne & Minimum & Maximum & Coefficient de variation (\%) \\
\hline Àge (années) & 63 & 26 & 105 & 59,2 \\
Nombre de tiges par hectare & 569 & 200 & 2600 & 72,6 \\
Circonférence moyenne $(\mathrm{cm})$ & 101 & 40 & 160 & 25,7 \\
Volume (m $3 /$ ha) & 461 & 249 & 677 & 18,2 \\
Hauteur dominante $(\mathrm{m})$ & 26,9 & 14,6 & 35,8 & 15,7 \\
Site Index $(\mathrm{m})$ & 20,3 & 5,9 & 37,6 & 34,7 \\
\hline
\end{tabular}


itératives utilisées pour l'estimation des paramètres convergent rapidement vers une solution. Les paramètres $m$ et $\sigma$ de la distribution normale ont également été estimés pour ces mêmes placettes.

L'indice $e^{\prime}$ (équation [19]) a été défini pour chaque modalité d'estimation des paramètres de la distribution de Weibull et pour la distribution normale. Deux systèmes de classification par catégories de grosseur ont été envisagés: des classes de circonférence de $10 \mathrm{~cm}$ d'amplitude et des classes correspondant aux catégories commerciales en vigueur pour l'épicéa (catégories commerciales utilisées, de circonférences à $1,5 \mathrm{~m}$ : moins de 40,40-69, $70-89,90-119,120-149,150-179,180$ et plus). Les colonnes 1 et 2 du tableau II donnent les valeurs moyennes de $e^{\prime}$ pour chaque modalité d'estimation et pour chaque type de classes de grosseur.

Une analyse de la variance de $e^{\prime}$ à 2 critères (méthode d'estimation et placette) fait apparaître des différences significatives, voire hautement significatives, entre les méthodes d'estimations des distributions théoriques, quel que soit le type de classes de grosseurs envisagé. Dans les 2 cas, la distribution de Weibull estimée par les moments non centrés ainsi que la dis- tribution normale donnent les meilleurs résultats.

\section{Prédiction des paramètres}

Pour prédire les paramètres de Weibull résultant d'une des 3 méthodes proposées, et ceux de la distribution normale, nous avons cherché à ajuster, aux valeurs des paramètres estimées pour les 141 placettes, des équations mettant en œuvre les variables définies au tableau I, ainsi que les variables résultant de la transformation de ces dernières par application des opérateurs $\log (),()^{2}$ et ()$^{0,5}$. Une procédure progressive (stepwise) a été utilisée pour définir les équations présentant une variabilité résiduelle minimale tout en affichant des distributions de résidus acceptables (tableau III).

D'une manière générale, on observe que, d'une part, seules les variables cmoy et age interviennent dans la prédiction des paramètres et que, d'autre part, la variabilité résiduelle est importante, voire très importante, dans tous les cas.

L'utilisation de ces équations a permis de prédire la distribution des tiges pour les

Tableau II. Valeurs moyennes des indices $e^{\prime}{ }_{1}$ (classes de $10 \mathrm{~cm}$ d'amplitude) et $e_{2}^{\prime}$ (classes "marchandes" pour chaque méthode de construction de la distribution théorique).

Méthodes Estimation des paramètres Prédiction des paramètres

\begin{tabular}{lllll}
\hline Weibull & $\mathrm{e}_{1}^{\prime}(\%)$ & $\mathrm{e}_{2}^{\prime}(\%)$ & $\mathrm{e}_{1}^{\prime}(\%)$ & $\mathrm{e}_{2}{ }_{2}(\%)$ \\
$\quad$ Maximum de vraisemblance & 32,0 & 14,2 & 36,6 & 21,2 \\
Moments non centrés & 30,2 & 12,5 & 34,4 & 18,8 \\
$\quad$ Moments pondérés & 33,9 & 15,5 & 37,4 & 23,6 \\
Normale & 31,7 & 13,9 & 34,1 & 17,7 \\
Analyses de la variance $(\alpha)$ & 0,004 & 0,000 & 0,000 & 0,000 \\
\hline
\end{tabular}

Les colonnes 1 et 2 correspondent à l'estimation et les colonnes 3 et 4 à la prédiction des paramètres. Le résultat des analyses de la variance est donné par la valeur du risque de première espèce $(\alpha)$. 
Tableau III. Équations de régressions retenues pour la prédiction des paramètres des distributions théoriques relatifs à chaque méthode.

\begin{tabular}{|c|c|c|c|}
\hline Méthodes & Équations & $R^{2}$ & $C V_{r}$ \\
\hline \multicolumn{4}{|l|}{ Weibull } \\
\hline \multirow[t]{3}{*}{ Maximum de vraisemblance } & $\mathrm{a}=14,96+0,003337$. cmoy $^{2}$ & 0,49 & 0,35 \\
\hline & $\mathrm{b}=-16,68+7,224$. cmoy $^{0,5}$ & 0,19 & 0,35 \\
\hline & $c=2,197+0,0128$. age & 0,05 & 0,31 \\
\hline \multirow{3}{*}{ Moments non centrés } & $a=-6,532+0,4503$. cmoy & 0,17 & 0,65 \\
\hline & $\mathrm{b}=7,917+0,5999 . \mathrm{cmoy}$ & 0,25 & 0,39 \\
\hline & $\mathrm{C}=1,887+0,3159$. age & 0,08 & 0,51 \\
\hline \multirow{3}{*}{ Moments pondérés } & $\mathrm{a}=14,34+0,002094 . \mathrm{cmoy}^{2}$ & 0,15 & 0,70 \\
\hline & $\mathrm{b}=-33,13+10,13$. cmoy 0.5 & 0,22 & 0,40 \\
\hline & $c=2,047+0,3460$. age & 0,03 & 0,70 \\
\hline \multirow[t]{2}{*}{ Normale } & cmoy $=$ cmoy & - & - \\
\hline & $s=\exp (0,5183+0,5156 \cdot \log (\mathrm{cmoy}))$ & 0,46 & 0,05 \\
\hline
\end{tabular}

cmoy représente la circonférence moyenne (en $\mathrm{cm}$ ) et age. l'âge du peuplement (en années). $\mathrm{R}^{2}$ est le coefficient de détermination et $\mathrm{CV}_{r}$, le coefficient de variation résiduelle.

141 placettes et de calculer ainsi l'indice $e^{\prime}$ pour les 2 classifications déjà utilisées au paragraphe précédent $\left(e^{\prime}{ }_{1}\right.$ pour les classes de $10 \mathrm{~cm}$ et $e_{2}^{\prime}$ pour les classes "marchandes"). Les colonnes 3 et 4 du tableau II donnent les valeurs moyennes de ces indices pour les différentes méthodes.

Les analyses de la variance opérées sur ces données démontrent que, quelles que soient les classes de grosseur envisagées, il existe des différences hautement significatives entre les méthodes de prédiction des distributions théoriques. Comme dans le cas de l'estimation des paramètres, la distribution normale ainsi que la distribution de Weibull définie par les moments non centrés ont donné les meilleurs résultats.

La dimension des classes utilisées pour la répartition des tiges influence fort logiquement la valeur de l'indice $e^{\prime}$. Si l'on considère l'ensemble des méthodes étudiées, celui-ci diminue de $32,0 \%$ à $14,0 \%$ dans le cas de l'estimation des paramètres et de $35,6 \%$ à $20,3 \%$ dans le cas de la prédiction des paramètres, quand on passe de la classification décimétrique à la classification commerciale plus grossière.

II est intéressant de noter également que, dans le cas des classes de $10 \mathrm{~cm}$ d'amplitude, malgré la faible efficacité des équations de régressions $\left(R^{2}<0,50\right)$, la part de l'imprécision des modèles liée à la phase de prédiction des paramètres est beaucoup moins importante que celle qui découle de la phase d'estimation. Si l'on considère l'ensemble des méthodes, on passe en effet d'un indice $e^{\prime}$ moyen de $32,0 \%$ (pour l'estimation) à $35,6 \%$ (pour la prédiction), soit une augmentation de $3,6 \%$. L'augmentation est un peu plus élevée dans le cas des classes "marchandes" pour lesquelles on passe de $14,0 \%$ (pour l'estimation) à 20,3\% (pour la prédiction) soit une augmentation de 6,3\%.

Cette observation nous conduit à penser que la plus grande part de l'imprécision du modèle de répartition des tiges trouve son origine dans l'estimation des paramètres.

La figure 2 qui représente l'évolution de l'indice $e^{\prime}$, relatif à la phase d'estimation (valeurs moyennes) en fonction de l'effectif 
des échantillons confirme cette hypothèse. La précision de l'estimation des paramètres apparaît étroitement liée à l'effectif de l'échantillon contenu dans la placette, quelle que soit la méthode utilisée.

Le modèle mettant en œuvre la distribution normale nécessite la connaissance d'une seule variable qui est la circonférence moyenne. L'utilisation de ce modèle permet une représentation tabulaire simple de la répartition des tiges par classes de grosseur pour différentes valeurs de la circonférence moyenne (tableau IV). En outre, I'utilisation conjointe d'un tarif de cubage à une entrée permet de présenter sous la même forme la répartition du volume par classes de grosseur (tableau V).

\section{EXEMPLE D'UTILISATION DU MODĖLE DE RÉPARTITION DES TIGES PAR CLASSES DE GROSSEURS}

L'utilisation d'un modèle de répartition des tiges doit s'envisager en complément d'un modèle plus général, tel qu'une table de production classique. Celle-ci est à même de fournir les informations permettant de définir les paramètres de la distribution théorique utilisée.

Dans l'exemple qui va suivre, nous utiliserons les données relatives à l'inventaire complet d'un peuplement, de manière à comparer les valeurs fournies par les différents modèles aux effectifs réellement observés. II s'agit d'un peuplement d'épicéa commun dont les caractéristiques sont les suivantes:

- âge : 55 ans;

- surface : 1,92 ha;

- circonférence moyenne : $119 \mathrm{~cm}$;

- nombre de tiges par ha : 380 .

Le tableau $V$ contient les effectifs observés et les effectifs estimés par les 4 méthodes présentées auparavant. Les va-

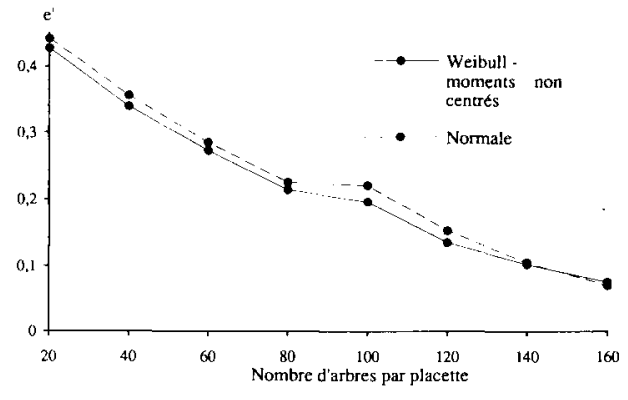

Fig 2. Évolution de l'indice $e^{\prime}{ }_{1}$ relatif à l'estimation des paramètres (valeurs moyennes pour des classes de $10 \mathrm{~cm}$ d'amplitude) en fonction de l'effectif des placettes.

leurs de l'indice $e^{\prime}$ sont également données pour chaque méthode et pour les 2 types de classes de grosseurs. Si l'on considère des classes de $10 \mathrm{~cm}$ d'amplitude, les différents modèles de distribution présentent des erreurs de distribution $\left(e_{1}^{\prime}\right)$ qui vont de $10,6 \%$ pour le modèle Weibull - maximum de vraisemblance à $18,2 \%$ pour le modèle Weibull - moments pondérés. Le modèle utilisant la distribution normale présente des performances comparables au premier $\left(e_{1}^{\prime}=11,6 \%\right)$.

\section{CONCLUSIONS}

Dans cette étude, nous avons envisagé la construction d'un modèle de répartition des grosseurs d'arbres pour des plantations d'épicéa commun.

Deux types de distributions théoriques ont été testées : la distribution de Weibull dont les paramètres ont été estimés par 3 méthodes différentes (maximum de vraisemblance, moments non centrés et moments pondérés) et la distribution normale.

La qualité des différents modèles testés a été appréciée, tant en phase d'estimation 


\begin{tabular}{|c|c|c|}
\hline & $\approx$ & $000000000000000000000000-$ \\
\hline & ה & $0000000000000000000000-N m$ \\
\hline & $\frac{n}{n}$ & $000000000000000000000-m n 9$ \\
\hline & 气े & $0000000000000000000-n+a, n$ \\
\hline & $\approx$ & 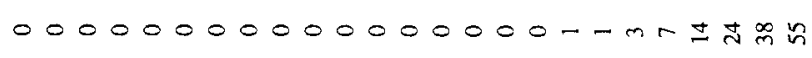 \\
\hline & $\infty$ & $0000000000000000-m 0 \Omega \pi m n ! \&$ \\
\hline & $\cong$ & 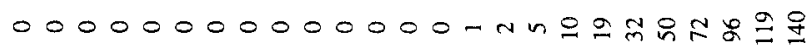 \\
\hline & $\underline{2}$ & $0000000000000-m \infty 1 \Omega$ F \\
\hline$\widehat{E}$ & 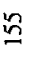 & 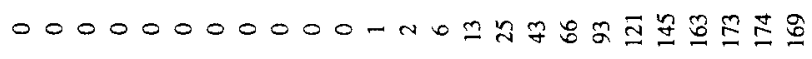 \\
\hline $\begin{array}{l}3 \\
\text { 离 } \\
\vdots \\
0\end{array}$ & 骂 & 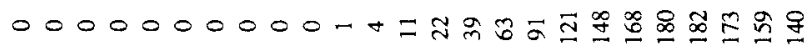 \\
\hline \& & $\stackrel{n}{m}$ & 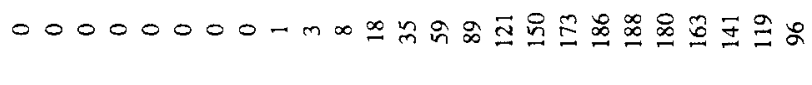 \\
\hline & $\dddot{y}$ & 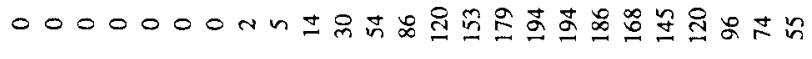 \\
\hline & $\cong$ & 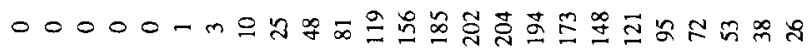 \\
\hline & $\stackrel{2}{\varrho}$ & 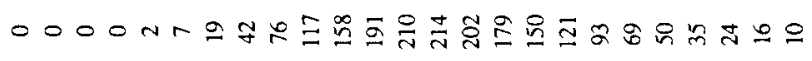 \\
\hline & 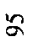 & 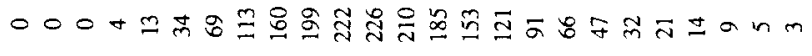 \\
\hline & $\infty$ & 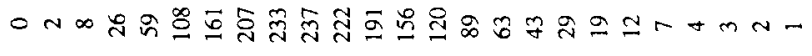 \\
\hline & $\cong$ & 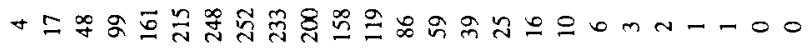 \\
\hline & $n$ & 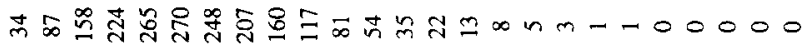 \\
\hline & $\curvearrowleft$ & 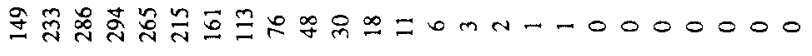 \\
\hline & $\approx$ & 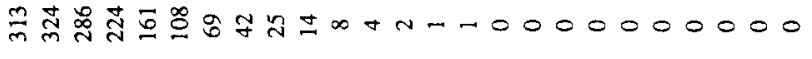 \\
\hline & $\approx$ & 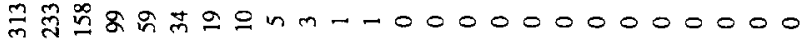 \\
\hline & $\sqrt{2}$ & 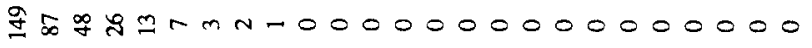 \\
\hline & $\because$ & DI $=0+n-0000000000000000000$ \\
\hline & 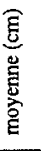 & 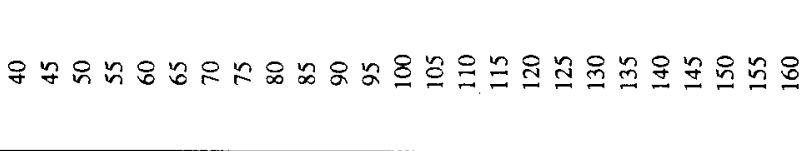 \\
\hline
\end{tabular}




\begin{tabular}{|c|c|}
\hline$\tilde{z}$ & $00000000000000000000000-n$ \\
\hline$\tilde{g}$ & $000000000000000000000-n m-$ \\
\hline$\frac{1}{6}$ & $00000000000000000000-m 0=\Omega$ \\
\hline$\check{c}$ & $000000000000000000-N n a=\infty \%$ \\
\hline$\dddot{g}$ & $0000000000000000-m \infty n \pi=8 \approx$ \\
\hline$\because$ & 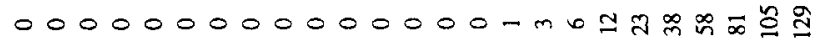 \\
\hline$\underline{7}$ & 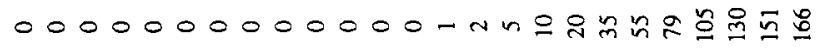 \\
\hline$y$ & 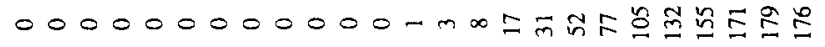 \\
\hline$\widehat{E}$ & 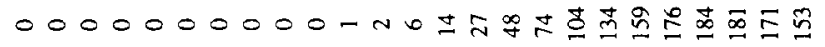 \\
\hline 鸢 & 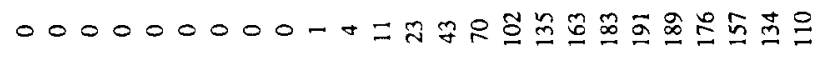 \\
\hline$y_{0}$ & 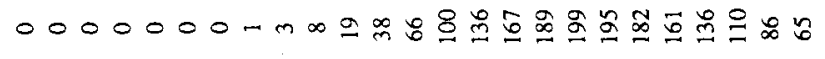 \\
\hline$\tilde{J}^{\infty}$ & 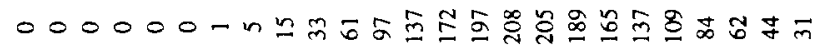 \\
\hline 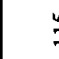 & 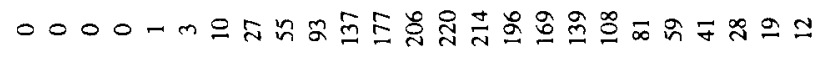 \\
\hline 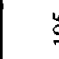 & 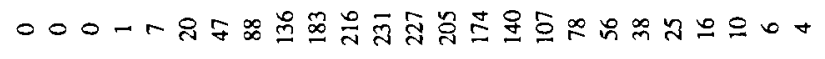 \\
\hline$y$ & 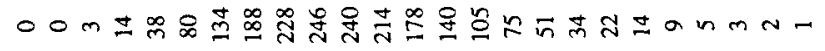 \\
\hline 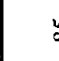 & - \\
\hline 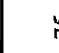 & ニ \\
\hline$y$ & 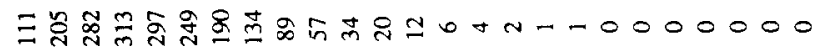 \\
\hline 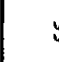 & 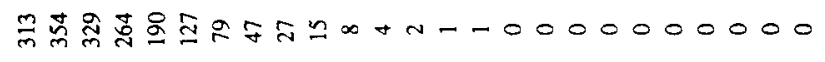 \\
\hline 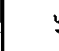 & 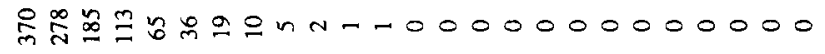 \\
\hline s. & $\because \&$ \& $\because=n n-00000000000000000$ \\
\hline 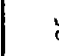 & $\pi 0+n-00000000000000000000$ \\
\hline 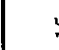 & -000000000000000000000000 \\
\hline 总 & 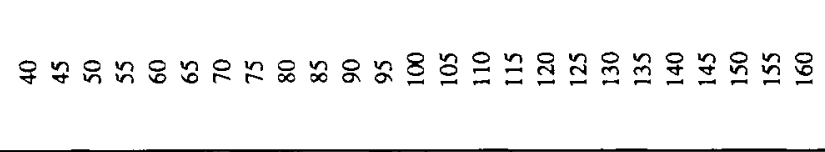 \\
\hline
\end{tabular}


Tableau VI. Effectifs observés et estimés pour un peuplement de 1,92 ha âgé de 55 ans.

\begin{tabular}{|c|c|c|c|c|c|}
\hline \multirow{2}{*}{$\begin{array}{c}\text { Classes } \\
\text { de grosseurs }\end{array}$} & \multirow{2}{*}{$\begin{array}{l}\text { Effectifs } \\
\text { observés }\end{array}$} & \multicolumn{4}{|c|}{ Effectifs estimés } \\
\hline & & $\begin{array}{c}\text { Weibull } \\
\text { maximum de } \\
\text { vraisemblance }\end{array}$ & $\begin{array}{c}\text { Weibull } \\
\text { moments } \\
\text { non centrés }\end{array}$ & $\begin{array}{l}\text { Weibull } \\
\text { moments } \\
\text { pondérés }\end{array}$ & Normale \\
\hline 55 & 0 & 0 & 1 & 1 & 1 \\
\hline 65 & 0 & 2 & 7 & 8 & 5 \\
\hline 75 & 4 & 16 & 21 & 24 & 15 \\
\hline 85 & 42 & 47 & 44 & 52 & 36 \\
\hline 95 & 88 & 84 & 75 & 88 & 70 \\
\hline 105 & 102 & 117 & 105 & 123 & 109 \\
\hline 115 & 138 & 132 & 124 & 140 & 135 \\
\hline 125 & 142 & 124 & 124 & 128 & 133 \\
\hline 135 & 94 & 96 & 102 & 92 & 105 \\
\hline 145 & 57 & 61 & 69 & 49 & 66 \\
\hline 155 & 36 & 32 & 37 & 19 & 33 \\
\hline 165 & 14 & 13 & 15 & 5 & 13 \\
\hline 175 & 9 & 4 & 5 & 1 & 4 \\
\hline 185 & 3 & 1 & 1 & 0 & 1 \\
\hline$e^{\prime 1}$ & & 0,106 & 0,133 & 0,182 & 0,116 \\
\hline$e^{\prime 2}$ & & 0,062 & 0,064 & 0,182 & 0,068 \\
\hline
\end{tabular}

Valeurs de l'indice $e^{\prime}$ pour les différents modèles de répartition et pour les 2 types de classes de grosseurs $\left(e_{1}^{\prime}\right.$ pour les classes de $10 \mathrm{~cm}$ d'amplitude et $e_{2}^{\prime}$ pour les classes "marchandes").

qu'en phase de prédiction à l'aide d'un indice proposé par Reynolds et al (1988).

Sur la base des données dont nous disposions (141 placettes de 10 ares), la distribution normale a donné des résultats aussi satisfaisants que la distribution de Weibull, et ce malgré une flexibilité moindre. Pour cette dernière, la méthode d'estimation des paramètres par les moments non centrés s'est révélée être la meilleure.

Du fait de la mise en cuvre plus simple, la distribution normale semble devoir être retenue pour la construction du modèle final.

La phase d'estimation des paramètres constitue la source la plus importante d'imprécision dans la construction d'un modèle de répartition quelle que soit la distribution théorique retenue. Cette constatation est à mettre en relation avec la liaison étroite que l'on observe entre la précision de cette estimation et le nombre d'individus présents dans les différents échantillons.

La construction des équations de prédiction des paramètres à partir de données "peu précises" explique les coefficients de détermination assez bas que l'on a obtenus.

Semblable étude devrait pouvoir être reconduite en disposant d'échantillons beaucoup plus étoffés (au moins une centaine d'arbres par placette), ce qui permettrait de juger les capacités réelles des 2 types de distribution à représenter les peuplements pour lesquels on aurait une image suffisamment représentative de la structure. 


\section{RÉFÉRENCES}

Bailey RL, Dell TR (1973) Quantifying diameter distributions with the Weibull function. Forest Sci 19 (2), 97-104

Borders BE, Souter RA, Bailey RI, Ware KD (1987) Percentile-based distributions characterize forest stand tables. Forest Sci 33 (2), 570-576

Burk TE, Newberry JD (1984) A simple algorithm for moment-based recovery of Weibull distribution parameters. Forest Sci 30 (2), 329-332

Cao QV, Burkhart HE (1984) A segmented distribution approach for modeling diameter frequency data. Forest Sci 30 (1), 129-137

Dagnelie P (1968) À propos de l'emploi du test de Kolmogorov-Smirnov comme test de normalité. Biom Praxim 9(1), 3-13

Dagnelie $P$ (1975) Théorie et méthodes statistiques. Gembloux, Presses Agronomiques, vol $2,463 p$

Dagnelie P, Palm R, Rondeux J, Thill A (1988) Tables de production relatives à l'épicéa commun. Gembloux, Presses Agronomiques, $123 p$

Gérard $P$ (1975) Estimation en valeur des pessières. Bull Soc Roy For Belg 82 (4), 189-207

Grender JM, Dell TR, Reich RM (1990) Theory and derivation for Weibull parameter probability weighted moment estimators. USDA For Serv Res Pap SO-260, $19 p$

Houllier F, Bouchon J, Birot $Y$ (1991) Modélisation de la dynamique des peuplements forestiers : états et perspectives. Rev For $\mathrm{Fr} 43$ (2), 87-107

Hyink DM, Moser JW (1983) A generalized framework for projecting forest yield and stand structure using diameter distributions. Forest Sci 29 (1), 85-95

Johnson NL, Kotz S (1970) Distributions in statistics: continuous univariate distributions. Houghton Mifflin Co, Boston, vol 2, $306 p$

Knoebel BR, Burkhart HE, Beck DE (1986) A growth and yield model for thinned stands of Yellow-Poplar. Forest Sci 32 (2) sup Monograph 27, $62 p$

Laurent C, Rondeux J (1982) Étude comparative de diverses unités d'échantillonnage à surfaces prédéterminées (cas des forêts résineuses equiennes). Document 81-3. Centre de recherche et de promotion forestière, section "Aménagement et Production" (IRSIA), Fac Sci agron, Gembloux, $21 \mathrm{p}$

Munro DD (1974) Forest growth models - a prognosis. In: Growth models for tree and stand simulation. Research note $n^{\circ} 30$, Stockholm, Royal College of Forestry, $373 p$

Rennolls K, Geary DN, Rollinson JD (1985) Characterizing diameter distributions by the use of the Weibull distribution. For 58 (1), 5766

Reynolds MR, Burk TE, Huang WC (1988) Goodness-of-fit tests and model selection procedures for diameter distributions models. Forest Sci 34 (2), 373-399

Rondeux J (1973) Simulation de l'évolution de peuplements forestiers dans le contexte d'une sylviculture intensive. In: Growth models for tree and stand simulation. Research note $n^{\circ} 30$, Stockholm, Royal College of Forestry, $373 p$

Zarnoch SJ, Dell TR (1985) An evaluation of percentile and maximum likelihood estimators of Weibull parameters. Forest Sci 31 (1), 260-268 